\title{
Asymptomatic anterior shoulder dislocation of 24-year duration
}

\author{
F. Mancini $\cdot$ R. Postacchini $\cdot$ R. Caterini
}

Received: 15 September 2007/ Accepted: 29 February 2008/Published online: 28 May 2008

(C) Springer-Verlag 2008

\begin{abstract}
A 73-year-old woman presented with a very long-standing anterior dislocation of her right shoulder. She had no pain, mild impairment of active shoulder motion and clinical features suggesting no tear of the rotator cuff. CT 3D reconstructions showed a newly formed glenoid cavity below the coracoid process. This case indicates that an anterior shoulder dislocation lasting even decades may be compatible with an almost normal shoulder function.
\end{abstract}

Keywords Shoulder · Long-standing dislocation . Glenoid

\section{Introduction}

Anterior shoulder dislocation is a common condition, responsible for severe pain and functional impairment. Almost consistently the dislocation is diagnosed and reduced in the emergency room. However, chronic unreduced dislocation, first reported by White [1] in 1741, may occasionally be encountered. The term chronic, although used even for dislocations of a few days duration [2], should be applied to dislocations lasting 3 or more weeks [3] or at least 4 weeks [4].

It is generally believed that in chronic dislocations surgical management should be carried out because no treatment leads to poor results, although it is also known that even surgery may give unsatisfactory results.

F. Mancini $\cdot$ R. Postacchini $(\bowtie) \cdot$ R. Caterini

Department of Orthopaedic Surgery, University "Tor Vergata",

Via g. Curioni, 99, 00157 Rome, Italy

e-mail: robby1478@hotmail.com
However, little information is available on the long-term natural history of unreduced anterior dislocations.

We report the case of a 73-year-old woman with an anterior dislocation of the right shoulder lasting as long as 24 years, who had no significant pain and very mild functional disability.

\section{Case report}

In January 2007, a 73-year-old woman, farm worker, was admitted to the Neurological Department of our hospital due to a transient ischemic attack of mild severity. The neurologists noticed that her shoulder profile was abnormal and thus asked an orthopaedic consultation.

When seen by one of us, the patient reported that at 49 years she had sustained a severe trauma on her right shoulder following a fall from a tree. She had severe pain for about 1 month and functional impairment for one more month. Subsequently, functional limitation gradually decreased and she returned to her usual activities. She had never sought an orthopaedic examination in her life.

On physical examination, the right shoulder region showed a prominence of the lateral border of the acromion, moderate atrophy of the deltoid and a bone tumefaction below the coracoid process (Fig. 1a). The passive forward flexion and abduction of her right arm were within normal limits, whereas external rotation was $40^{\circ}$ and internal posterior rotation was limited to L5. The active forward flexion was $170^{\circ}$, and the active abduction and external rotation were $120^{\circ}$ and $30^{\circ}$, respectively, thus allowing her right hand to reach the occiput (Fig. 1b, c). The posterior internal rotation, instead, was significantly impaired (Fig. 1d). The strength of external rotation and abduction of the arm against the resistance of the examiner's hand 
was only slightly less than on the opposite side and the belly press test was negative, thus suggesting that the rotator cuff was intact. The right biceps muscle was atrophic, suggesting an old standing tear of the bicipital tendon. The Constant score was 78 .

$\mathrm{X}$-rays and CT scan with 3D reconstructions of the right shoulder were carried out, while MRI could not be obtained because the patient had a pace-maker. CT reconstruction showed an anterior dislocation of the right humeral head, which had an irregular surface (Fig. 2a). A defect was visible in the centre of the anterior border of the natural glenoid cavity as a result of a glenoid fracture or bone wear caused by the humeral head (Fig. 2b). The latter was in contact with a cavity located in the body of the scapula under the coracoid process (Fig. 2c). The cavity, which had a vertical orientation and was slightly less deep than the original glenoid, had the appearance of a newly formed glenoid. On axial CT scans, the newly formed glenoid had a $45^{\circ}$ orientation with respect to the horizontal plane (Fig. 2d). Its surface was smooth and the underlying bone was sclerotic. The humeral head was severely osteoporotic and the portion of its surface facing the newly formed glenoid was fairly smooth.

\section{Discussion}

Chronic shoulder dislocation is an uncommon condition. Rowe and Zarins [3] evaluated the results of treatment in 23 shoulder dislocations that had gone unreduced for at least 3 weeks. Eight dislocations were anterior and 14 were posterior. In seven of the patients with an anterior dislocation, the duration of disability ranged from 3 weeks to 2 years, while in one case the dislocation was recognized 10 years after injury. Goga [2] reported on 32 patients with chronic shoulder dislocation diagnosed over a 5 -year period. In the 31 cases with an anterior dislocation the longest period of failed recognition of the condition was 2 years. Postacchini and Facchini [5], in a series of 12 cases with an unreduced shoulder dislocation, found 5 patients with an anterior dislocation lasting no more than 6 weeks; one patient in the latter group, who refused either manipulation or surgery, was found to have had a spontaneous reduction of the dislocation at 6-year follow-up. Mansat et al. [6] operated on five patients in whom an anterior shoulder dislocation had been unrecognized from 6 weeks to 3 years. In our case the duration of shoulder dislocation is by far the longest ever reported.
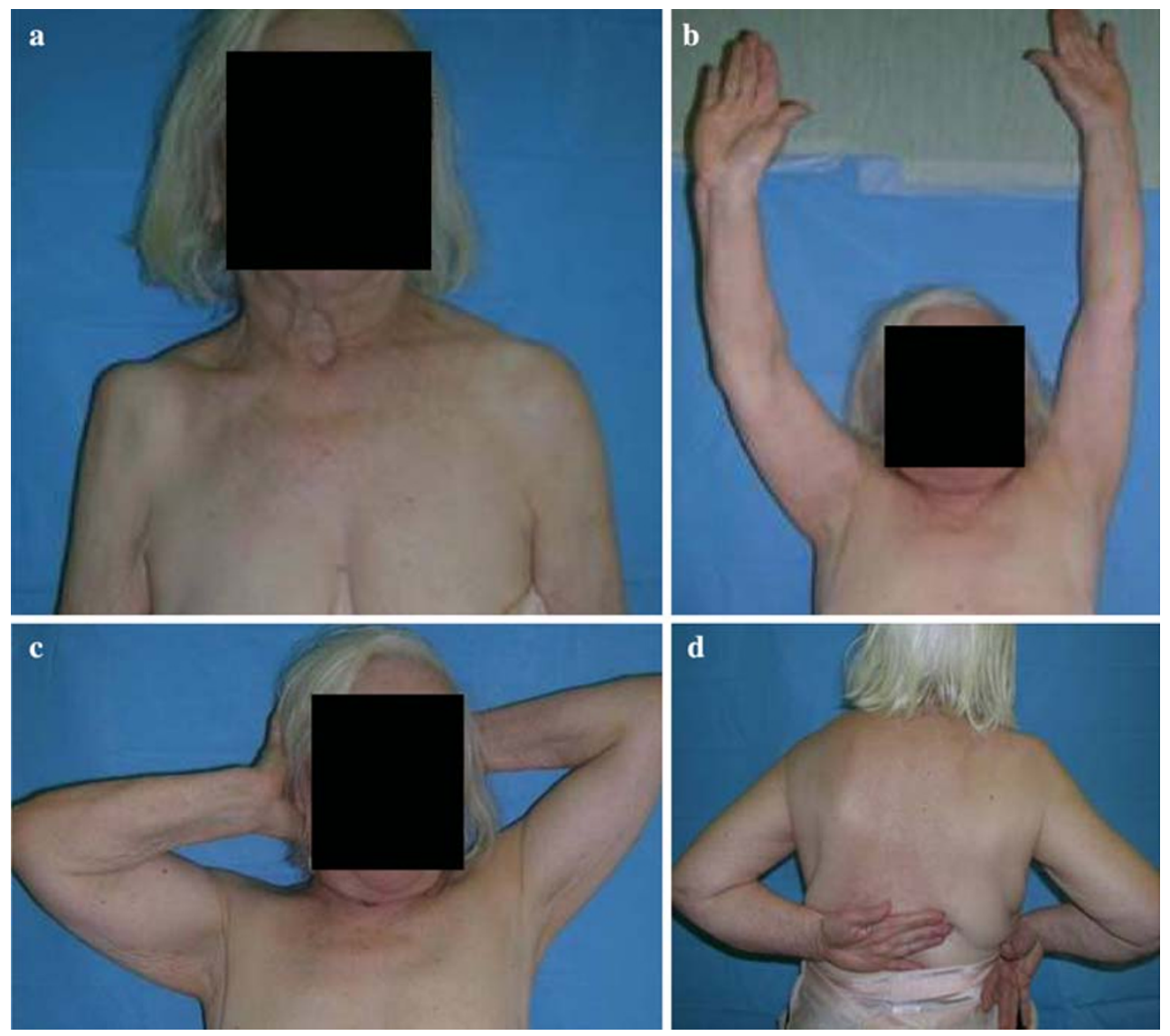

Fig. 1 Photographs of the patient with chronic unreduced shoulder dislocation showing, on the right side, a tumefaction below the coracoid process and prominence of the lateral border of the acromion (a), full forward flexion (b), almost normal abduction-external rotation $(\mathbf{c})$ and limited internal rotation $(\mathbf{d})$ of the arm 

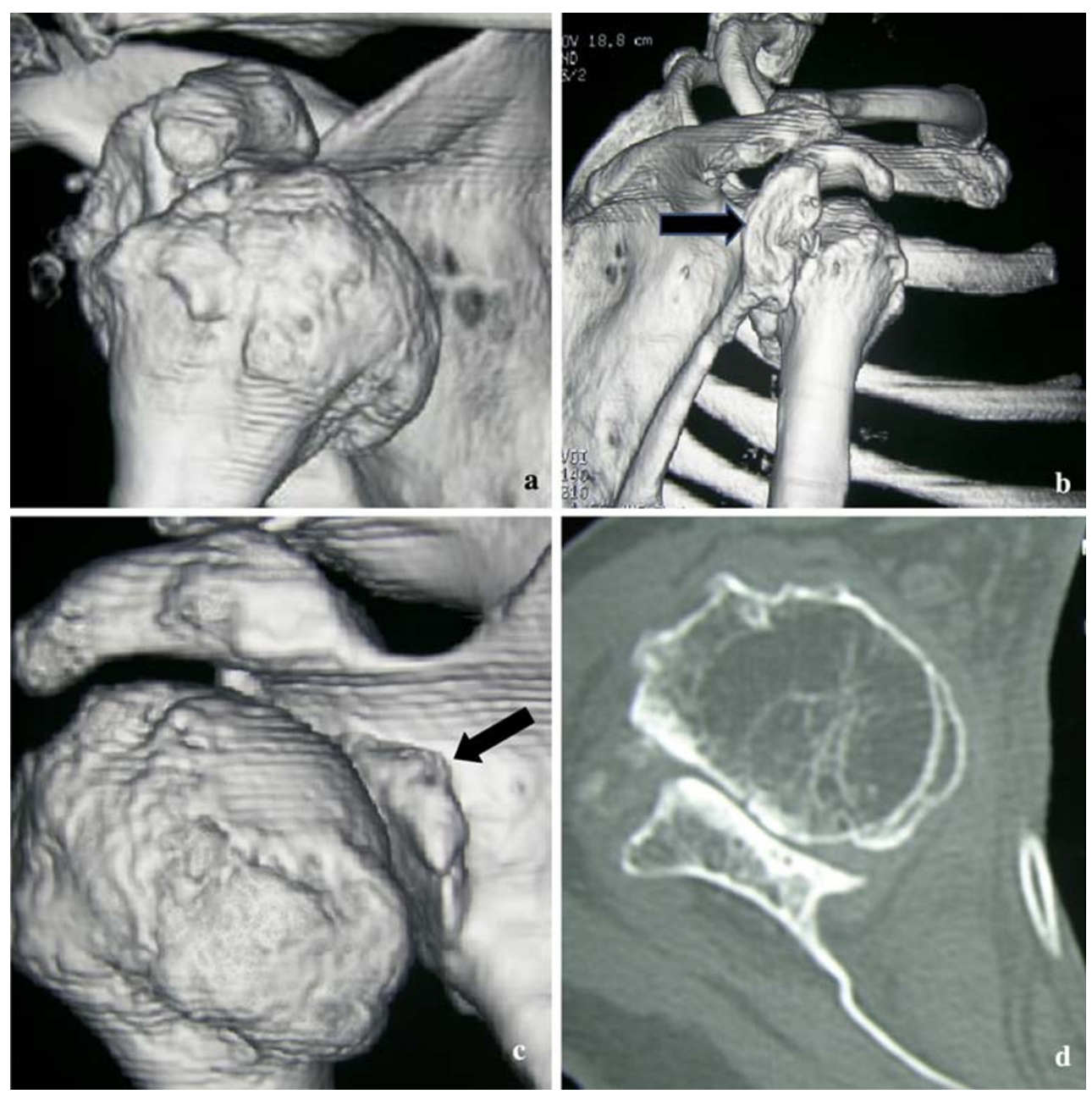

Fig. 2 The 3D CT scans show the right humeral head dislocated anteriorly below the coracoid (a), the empty original glenoid cavity (arrow) with an irregular surface and an anterior bone defect (b), and a newly formed glenoid cavity (arrow) in contact with the humeral

Treatment options for chronic shoulder dislocation include no treatment, open reduction with preservation of the humeral head, resection of the humeral head (Jones procedure) and hemiarthroplasty.

Manipulation may be successful only in dislocations of few weeks duration $[2,3,5,7]$. Open reduction have given satisfactory results, but in most cases it is indicated in patients whose dislocation lasts not more than several months $[3,6]$. Resection of the humeral head was found to give unexpectedly good results, although nowadays it is considered as an unacceptable procedure. Hemiarthroplasty or total shoulder arthroplasty have been used only in few patients with results varying from good to poor $[3,8]$. Goga [2] found that the patients treated surgically by open reduction fared much better that those left untreated. Nevertheless, several authors [7, 9-12] advocate no treatment in elderly patients who do not have disabling pain and function or in the presence of very long-standing head (c). On the axial CT scan (d), the newly formed glenoid, showing a smooth surface, has a $45^{\circ}$ orientation with respect to the horizontal plane and the humeral head appears severely osteoporotic

dislocation. In our patient we did not attempt any treatment because of the satisfactory clinical status and the extremely long duration of the dislocation.

The present case may be useful to better understand the long-term natural history of chronic anterior shoulder dislocation. The satisfactory result found in our patient may be related to the presence of a newly formed glenoid cavity in the body of the scapula, a finding never reported in the literature. However, also the rotator cuff, which appeared to be intact, may have played a role. It is known, in fact, that elderly patients undergoing anterior shoulder dislocation very often have a concomitant cuff tear [13], whereas in subjects younger than 50 years, as was our patient at the time of injury, no cuff rupture usually occurs. The bicipital tendon, however, was most probably torn. This may have occurred at the time of dislocation or subsequently due to attrition against the displaced bicipital groove. 
Conflict of interest statement The authors declare that they have no conflict of interest related to the publication of this manuscript.

Consent to publication The patient gave informed consent to the publication of this case report.

\section{References}

1. White, Charles Essays in surgery (1741)

2. Goga IE (2003) Chronic shoulder dislocations. J Shoulder Elbow Surg 12(5):446-450

3. Rowe CR, Zarins B (1982) Chronic unreduced dislocations of the shoulder. J Bone Joint Surg Am 64(4):494-505

4. Souchon E (1891) Operative treatment of irreducible dislocation of the shoulder joint, recent or old, simple or complicated. Trans Am Surg Assoc 15:311-442

5. Postacchini F, Facchini M (1987) The treatment of unreduced dislocation of the shoulder. A review of 12 cases. Ital J Orthop Traumatol 13(1):15-26
6. Mansat P, Guity MR, Mansat M, Bellumore Y, Rongieres M, Bonnevialle P (2003) Chronic anterior shoulder dislocation treated by open reduction sparing the humeral head. Rev Chir Orthop Reparatrice Appar Mot 89(1):19-26

7. Schulz TJ Jr (1969) Urecognized dislocation of the shoulder. J Trauma 9:1009-1023

8. Pritchett JW, Clark JM (1987) Prosthetic replacement for chronic unreduced dislocations of the shoulder. Clin Orthop Relat Res 216:89-93

9. Noack W, Strohmeier M (1988) Treatment of unreduced anterior shoulder dislocation. Unfallchirurgie 14(4):184-190

10. Bennet GE (1936) Old dislocation of the shoulder. J Bone Joint Surg 18:594-606

11. Mosely HF (1969) Shoulder lesions. 3rd edn. Livingstone, Edimburgh pp. 155-161

12. Key JA (1946) Quoted by: Steinderl A. Traumatic deformities and disabilities of the upper extremity. Charles C. Thomas, Springfield pp 155-161

13. Postacchini F, Gumina S (1997) Anterior dislocation of the shoulder in elderly patients. J Bone Joint Surg Br 79(4):540-543 